\title{
Numerical Solutions of Fractional Fokker-Planck Equations Using Iterative Laplace Transform Method
}

\author{
Limei Yan \\ School of Mathematical Sciences, Dezhou University, Dezhou 253023, China \\ Correspondence should be addressed to Limei Yan; yanlimei2013@163.com
}

Received 3 August 2013; Revised 17 October 2013; Accepted 2 November 2013

Academic Editor: Dumitru Baleanu

Copyright (C) 2013 Limei Yan. This is an open access article distributed under the Creative Commons Attribution License, which permits unrestricted use, distribution, and reproduction in any medium, provided the original work is properly cited.

\begin{abstract}
A relatively new iterative Laplace transform method, which combines two methods; the iterative method and the Laplace transform method, is applied to obtain the numerical solutions of fractional Fokker-Planck equations. The method gives numerical solutions in the form of convergent series with easily computable components, requiring no linearization or small perturbation. The numerical results show that the approach is easy to implement and straightforward when applied to space-time fractional Fokker-Planck equations. The method provides a promising tool for solving space-time fractional partial differential equations.
\end{abstract}

\section{Introduction}

Fractional calculus has attracted much attention for its potential applications in various scientific fields such as fluid mechanics, biology, viscoelasticity, engineering, and other areas of science [1-4]. So it becomes important to find some efficient methods for solving fractional differential equations. A great deal of effort has been spent on constructing of the numerical solutions and many effective methods have been developed such as fractional wavelet method [5-8], fractional differential transform method [9], fractional operational matrix method $[10,11]$, fractional improved homotopy perturbation method $[12,13]$, fractional variational iteration method $[14,15]$, and fractional Laplace Adomian decomposition method $[16,17]$.

In 2006, Daftardar-Gejji and Jafari proposed a new iterative method to seek numerical solutions of nonlinear functional equations $[18,19]$. By now, the iterative method has been used to solve many nonlinear differential equations of integer and fractional order [20] and fractional boundary value problem [21]. Most recently, Jafari et al. firstly applied Laplace transform in the iterative method and proposed a new direct method called iterative Laplace transform method [22] to search for numerical solutions of a system of fractional partial differential equations. The method is based on Laplace transform, iterative method, Caputo fractional derivative, and symbolic computation. By using this method, Jafari and Seifi successfully obtained the numerical solutions of two systems of space-time fractional differential equations. It has been shown that, with this method, one can discover some solutions found by the existing methods such as homotopy perturbation method, Laplace Adomian decomposition method, and variational iterative method [23].

It is well known that the choice of an appropriate ansatz is of great importance when a method is applied to search for numerical solutions of nonlinear partial differential equations. In the present paper, we will use the iterative Laplace transform method to solve space-time fractional FokkerPlanck equations. The fractional derivatives described here are in the Caputo sense.

Fokker-Planck equation has been applied in various natural science fields such as quantum optics, solid-state physics, chemical physics, theoretical biology, and circuit theory. It is firstly proposed by Fokker and Planck to characterize the Brownian motion of particles [24]. The general form of Fokker-Planck equation is as follows:

$$
\frac{\partial u}{\partial t}=\left[-\frac{\partial}{\partial x} A(x)+\frac{\partial^{2}}{\partial x^{2}} B(x)\right] u(x, t)
$$

with the initial condition

$$
u(x, 0)=f(x),
$$


where $A(x), B(x)>0$ are called diffusion coefficient and drift coefficient, respectively. If $A(x), B(x)>0$ depend on $u(x, t)$ and the time $t$, then (1) becomes the following generalized nonlinear form [25]:

$$
\frac{\partial u}{\partial t}=\left[-\frac{\partial}{\partial x} A(x, t, u)+\frac{\partial^{2}}{\partial x^{2}} B(x, t, u)\right] u(x, t) .
$$
[26]:

The space-time fractional Fokker-Planck is as follows

$$
D_{t}^{\alpha} u=\left[-D_{x}^{\beta} A(x, t, u)+D_{x}^{2 \beta} B(x, t, u)\right] u(x, t)
$$

which is the generalized fractional form of (3). Here $D_{t}^{\alpha}(\cdot), D_{x}^{\beta}(\cdot), D_{x}^{2 \beta}(\cdot)$ are the Caputo fractional derivative with respect to $t$ and $x$ defined in Section 2. When $\alpha=\beta=1$, (4) reduces to (3).

The rest of this paper is organized as follows. In Section 2, we review some basic definitions of Caputo fractional derivative and Laplace transform. In Section 3, we describe the iterative Laplace transform method for solving fractional partial differential equations. In Section 4, we give three applications of the method to Fokker-Planck equations. In Section 5, some conclusions and discussions are given.

\section{Preliminaries}

Definition 1. The Caputo fractional derivative $[27,28]$ of function $u(x, t)$ is defined as

$$
\begin{array}{r}
D_{t}^{\alpha} u(x, t)=\frac{1}{\Gamma(m-\alpha)} \int_{0}^{t}(t-\eta)^{m-\alpha-1} u^{(m)}(x, \eta) d \eta, \\
m-1<\alpha \leq m, m \in N,
\end{array}
$$

where $\Gamma(\cdot)$ denotes the gamma function.

Definition 2. The Laplace transform of $f(t)$ is defined as [27, 28]

$$
F(s)=\mathscr{L}[f(t)]=\int_{0}^{\infty} e^{-s t} f(t) d t
$$

Definition 3. Laplace transform of $D_{t}^{\alpha} u(x, t)$ is given as [27, 28]

$$
\begin{array}{r}
\mathscr{L}\left[D_{t}^{\alpha} u(x, t)\right]=s^{\alpha} \mathscr{L}[u(x, t)]-\sum_{k=0}^{m-1} u^{(k)}(x, 0) s^{\alpha-1-k}, \\
m-1<\alpha \leq m,
\end{array}
$$

where $u^{(k)}(x, 0)$ is the $k$-order derivative of $u(x, t)$ at $t=0$.

Further information about fractional derivative and its properties can be found in [27-29].

\section{The Iterative Laplace Transform Method}

To illustrate the basic idea of the iterative Laplace transform method [22], we consider the general space-time fractional partial differential equation with initial conditions of the form

$$
\begin{array}{r}
D_{t}^{\alpha} u=\mathscr{A}\left(u, D_{x}^{\beta} u, D_{x}^{2 \beta} u, \ldots\right), \quad m-1<\alpha \leq m, \\
n-1<\beta \leq n, \quad m, n \in N,
\end{array}
$$

with initial value conditions

$$
u^{(k)}(x, 0)=h_{k}(x), \quad k=0,1, \ldots, m-1,
$$

where $\mathscr{A}\left(u, D_{x}^{\beta} u, D_{x}^{2 \beta} u, \ldots\right)$ is a linear or nonlinear operator of $u, D_{x}^{\beta} u, D_{x}^{2 \beta} u, \ldots$, and $u=u(x, t)$ is the unknown function that will be determined later.

Taking Laplace transfer of both sides of (8) results in

$$
\begin{gathered}
s^{\alpha} \mathscr{L}[u(x, t)]-\sum_{k=0}^{m-1} s^{\alpha-1-k} u^{(k)}(x, 0) \\
=\mathscr{L}\left[\mathscr{A}\left(u, D_{x}^{\beta} u, D_{x}^{2 \beta} u, \ldots\right)\right] .
\end{gathered}
$$

Equivalently,

$$
\begin{aligned}
\mathscr{L}[u(x, t)]= & \sum_{k=0}^{m-1} s^{-1-k} u^{(k)}(x, 0) \\
& +s^{-\alpha} \mathscr{L}\left[\mathscr{A}\left(u, D_{x}^{\beta} u, D_{x}^{2 \beta} u, \ldots\right)\right] .
\end{aligned}
$$

Operating with Laplace inverse (denoted by $\mathscr{L}^{-1}$ throughout the present paper) on both sides of (11) gives

$$
\begin{aligned}
u(x, t)= & \mathscr{L}^{-1}\left[\sum_{k=0}^{m-1} s^{-1-k} u^{(k)}(x, 0)\right] \\
& +\mathscr{L}^{-1}\left[s^{-\alpha} \mathscr{L}\left[\mathscr{A}\left(u, D_{x}^{\beta} u, D_{x}^{2 \beta} u, \ldots\right)\right]\right],
\end{aligned}
$$

which can be rewritten as the form

$$
\begin{aligned}
u(x, t)= & \mathscr{L}^{-1}\left[\sum_{k=0}^{m-1} s^{-1-k} u^{(k)}(x, 0)\right] \\
& +\mathscr{B}\left(u, D_{x}^{\beta} u, D_{x}^{2 \beta} u, \ldots\right)
\end{aligned}
$$

where $\mathscr{B}\left(u, D_{x}^{\beta} u, D_{x}^{2 \beta} u, \ldots\right)=\mathscr{L}^{-1}\left[s^{-\alpha} \mathscr{L}\left[\mathscr{A}\left(u, D_{x}^{\beta} u, D_{x}^{2 \beta} u\right.\right.\right.$, $\ldots)]$.

The iterative Laplace transform method represents the solution as an infinite series:

$$
u(x, t)=\sum_{n=0}^{\infty} u_{n}
$$


where the terms $u_{n}$ are to be recursively computed. The linear or nonlinear operator $\mathscr{B}\left(u, D_{x}^{\beta} u, D_{x}^{2 \beta} u, \ldots\right)$ can be decomposed as follows:

$$
\begin{aligned}
\mathscr{B}\left(\sum_{n=0}^{\infty} u_{n}, D_{x}^{\beta} \sum_{n=0}^{\infty} u_{n}, D_{x}^{2 \beta} \sum_{n=0}^{\infty} u_{n}, \ldots\right) \\
=\mathscr{B}\left(u_{0}, D_{x}^{\beta} u_{0}, D_{x}^{2 \beta} u_{0}, \ldots\right) \\
\quad+\sum_{j=1}^{\infty} \mathscr{B}\left(\sum_{k=0}^{j} u_{k}, D_{x}^{\beta} \sum_{k=0}^{j} u_{k}, D_{x}^{2 \beta} \sum_{k=0}^{j} u_{k}, \ldots\right) \\
\quad-\sum_{j=1}^{\infty} \mathscr{B}\left(\sum_{k=0}^{j-1} u_{k}, D_{x}^{\beta} \sum_{k=0}^{j-1} u_{k}, D_{x}^{2 \beta} \sum_{k=0}^{j-1} u_{k}, \ldots\right) .
\end{aligned}
$$

Substituting (14) and (15) into (13) yields

$$
\begin{aligned}
\sum_{n=0}^{\infty} u_{n}=\mathscr{L}^{-1}\left[\sum_{k=0}^{m-1} s^{-1-k} u^{(k)}(x, 0)\right] & \\
+ & \mathscr{B}\left(u_{0}, D_{x}^{\beta} u_{0}, D_{x}^{2 \beta} u_{0}, \ldots\right) \\
+ & \sum_{j=1}^{\infty}\left[\mathscr{B}\left(\sum_{k=0}^{j} u_{k}, D_{x}^{\beta} \sum_{k=0}^{j} u_{k}, D_{x}^{2 \beta} \sum_{k=0}^{j} u_{k}, \ldots\right)\right. \\
& \left.-\mathscr{B}\left(\sum_{k=0}^{j-1} u_{k}, D_{x}^{\beta} \sum_{k=0}^{j-1} u_{k}, D_{x}^{2 \beta} \sum_{k=0}^{j-1} u_{k}, \ldots\right)\right] .
\end{aligned}
$$

We set

$$
\begin{gathered}
u_{0}=\mathscr{L}^{-1}\left[\sum_{k=0}^{m-1} s^{-1-k} u^{(k)}(x, 0)\right], \\
u_{1}=\mathscr{B}\left(u_{0}, D_{x}^{\beta} u_{0}, D_{x}^{2 \beta} u_{0}, \ldots\right), \\
u_{m+1}=\mathscr{B}\left(\sum_{k=0}^{m} u_{k}, D_{x}^{\beta} \sum_{k=0}^{m} u_{k}, D_{x}^{2 \beta} \sum_{k=0}^{m} u_{k}, \ldots\right) \\
-\mathscr{B}\left(\sum_{k=0}^{m-1} u_{k}, D_{x}^{\beta} \sum_{k=0}^{m-1} u_{k}, D_{x}^{2 \beta} \sum_{k=0}^{m-1} u_{k}, \ldots\right), \quad m \geq 1 .
\end{gathered}
$$

Therefore the $m$-term numerical solution of (8)-(9) is given by

$$
\begin{array}{r}
u(x, t) \cong u_{0}(x, t)+u_{1}(x, t)+\cdots+u_{m}(x, t), \\
m=1,2, \ldots .
\end{array}
$$

\section{Numerical Solutions of Fractional Fokker-Planck Equations}

The iterative Laplace transform method, described in Section 3, will be applied to solve three special cases of space-time fractional Fokker-Planck equations with initial conditions.
Example 1. Consider the Fokker-Planck equation in the case that [30]

$$
D_{t}^{\alpha} u=\frac{\partial u}{\partial x}+\frac{\partial^{2} u}{\partial x^{2}}, \quad 0<\alpha \leq 1
$$

subject to

$$
u(x, 0)=x \text {. }
$$

Taking Laplace transform on both sides of (19) gives

$$
\begin{gathered}
s^{\alpha} \mathscr{L}[u(x, t)]-s^{\alpha-1} u(x, 0)=\mathscr{L}\left[\frac{\partial u}{\partial x}+\frac{\partial^{2} u}{\partial x^{2}}\right], \\
\mathscr{L}[u(x, t)]=\frac{x}{s}+\frac{1}{s^{\alpha}} \mathscr{L}\left[\frac{\partial u}{\partial x}+\frac{\partial^{2} u}{\partial x^{2}}\right] .
\end{gathered}
$$

Operating with Laplace inverse on both sides of (22) results in

$$
u(x, t)=x+\mathscr{L}^{-1}\left[\frac{1}{s^{\alpha}} \mathscr{L}\left[\frac{\partial u}{\partial x}+\frac{\partial^{2} u}{\partial x^{2}}\right]\right] .
$$

Substituting (14) and (15) into (23) and applying (17), we obtain the components of the solution as follows:

$$
\begin{gathered}
u_{0}(x, t)=u(x, 0)=x, \\
u_{1}(x, t)=\mathscr{L}^{-1}\left[\frac{1}{s^{\alpha}} \mathscr{L}\left[\frac{\partial u_{0}}{\partial x}+\frac{\partial^{2} u_{0}}{\partial x^{2}}\right]\right]=\frac{t^{\alpha}}{\Gamma(1+\alpha)}, \\
u_{2}(x, t)=0 \\
u_{3}(x, t)=\cdots=u_{n}(x, t)=\cdots=0 .
\end{gathered}
$$

Therefore, the solution of (19)-(20) in a closed form can be obtained as follows:

$$
u(x, t)=x+\frac{t^{\alpha}}{\Gamma(1+\alpha)} .
$$

If we take $\alpha=1$, then (25) can be reduced to

$$
u(x, t)=x+t,
$$

which is exactly the same as that obtained by homotopy perturbation method in [30].

It should be pointed out that the iterative Laplace transform method is the generalization algorithm of iterative method proposed by Daftardar-Gejji and Jafari [18]. When these two methods are used to solve differential equations with integer order derivatives, especially for linear cases, they are not different from each other.

Example 2. Consider the following space-time fractional Fokker-Planck equation with initial value conditions [26]:

$$
\begin{array}{r}
D_{t}^{\alpha} u=-D_{x}^{\beta}\left(\frac{u x}{6}\right)+D_{x}^{2 \beta}\left(\frac{u x^{2}}{12}\right), \\
t>0, x>0,0<\alpha, \beta \leq 1,
\end{array}
$$


subject to

$$
u(x, 0)=x^{2}
$$

Taking Laplace transform on both sides of (27) gives

$$
\begin{aligned}
& s^{\alpha} \mathscr{L}[u(x, t)]-s^{\alpha-1} u(x, 0) \\
& =\mathscr{L}\left[-D_{x}^{\beta}\left(\frac{u x}{6}\right)+D_{x}^{2 \beta}\left(\frac{u x^{2}}{12}\right)\right], \\
& \mathscr{L}[u(x, t)] \\
& =\frac{u(x, 0)}{s}+\frac{1}{s^{\alpha}} \mathscr{L}\left[-D_{x}^{\beta}\left(\frac{u x}{6}\right)+D_{x}^{2 \beta}\left(\frac{u x^{2}}{12}\right)\right] .
\end{aligned}
$$

Operating with Laplace inverse on both sides of (30), we obtain the following Laplace equation:

$$
\begin{aligned}
& u(x, t) \\
& \quad=u(x, 0)+\mathscr{L}^{-1}\left[\frac{1}{s^{\alpha}} \mathscr{L}\left[-D_{x}^{\beta}\left(\frac{u x}{6}\right)+D_{x}^{2 \beta}\left(\frac{u x^{2}}{12}\right)\right]\right] .
\end{aligned}
$$

Following the algorithm given in (17), the first three components of the solution are as follows:

$$
\begin{aligned}
& u_{0}(x, t)=u(x, 0)=x^{2}, \\
& u_{1}(x, t) \\
& =\mathscr{L}^{-1}\left[\frac{1}{s^{\alpha}} \mathscr{L}\left[-D_{x}^{\beta}\left(\frac{u_{0} x}{6}\right)+D_{x}^{2 \beta}\left(\frac{u_{0} x^{2}}{12}\right)\right]\right] \\
& =\left(-\frac{1}{\Gamma(4-\beta)} x^{3-\beta}+\frac{2}{\Gamma(5-2 \beta)} x^{4-2 \beta}\right) \cdot \frac{t^{\alpha}}{\Gamma(1+\alpha)} \text {, } \\
& u_{2}(x, t) \\
& =\left[\frac{4-\beta}{6 \Gamma(5-2 \beta)} x^{4-2 \beta}-\frac{5-3 \beta}{3 \Gamma(6-3 \beta)} x^{5-3 \beta}\right. \\
& -\frac{(5-\beta)(4-\beta)}{12 \Gamma(6-3 \beta)} x^{5-3 \beta}+\frac{(6-2 \beta)(5-2 \beta)}{6 \Gamma(7-4 \beta)} \\
& \left.\times x^{6-4 \beta}\right] \cdot \frac{t^{2 \alpha}}{\Gamma(1+2 \alpha)} \text {. }
\end{aligned}
$$

The solution in series form is then given by

$$
\begin{aligned}
& u(x, t) \\
& =x^{2}+\left(-\frac{1}{\Gamma(4-\beta)} x^{3-\beta}+\frac{2}{\Gamma(5-2 \beta)} x^{4-2 \beta}\right) \cdot \frac{t^{\alpha}}{\Gamma(1+\alpha)} \\
& +\left[\frac{4-\beta}{6 \Gamma(5-2 \beta)} x^{4-2 \beta}-\frac{5-3 \beta}{3 \Gamma(6-3 \beta)} x^{5-3 \beta}\right. \\
& \left.\quad-\frac{(5-\beta)(4-\beta)}{12 \Gamma(6-3 \beta)} x^{5-3 \beta}+\frac{(6-2 \beta)(5-2 \beta)}{6 \Gamma(7-4 \beta)} x^{6-4 \beta}\right] \\
& \cdot \frac{t^{2 \alpha}}{\Gamma(1+2 \alpha)}+\cdots .
\end{aligned}
$$

Setting $\alpha=\beta=1$ in (33), we get the solution of the problem by

$$
u(x, t)=x^{2}\left(1+\frac{t}{2}+\frac{(t / 2)^{2}}{2 !}+\cdots\right)
$$

and in a closed form by

$$
u(x, t)=x^{2} e^{t / 2}
$$

which is in full agreement with the results by homotopy perturbation method in [26].

Example 3. Consider the following space-time fractional nonlinear initial value problem that describes Fokker-Planck equation [12]:

$$
D_{t}^{\alpha} u=-D_{x}^{\beta}\left(\frac{4 u^{2}}{x}-\frac{x u}{3}\right)+D_{x}^{2 \beta} u^{2}, \quad 0<\alpha, \beta \leq 1,
$$

subject to

$$
u(x, 0)=x^{2}
$$

Taking Laplace transform on both sides of (35) gives

$$
\begin{gathered}
s^{\alpha} \mathscr{L}[u(x, t)]-s^{\alpha-1} u(x, 0) \\
=\mathscr{L}\left[-D_{x}^{\beta}\left(\frac{4 u^{2}}{x}-\frac{x u}{3}\right)+D_{x}^{2 \beta} u^{2}\right], \\
\mathscr{L}[u(x, t)]=\frac{x^{2}}{s}+\frac{1}{s^{\alpha}} \mathscr{L}\left[-D_{x}^{\beta}\left(\frac{4 u^{2}}{x}-\frac{x u}{3}\right)+D_{x}^{2 \beta} u^{2}\right] .
\end{gathered}
$$

Operating with Laplace inverse on both sides of (38), we obtain the following Laplace equation:

$$
u(x, t)=x^{2}+\mathscr{L}^{-1}\left[\frac{1}{s^{\alpha}} \mathscr{L}\left[-D_{x}^{\beta}\left(\frac{4 u^{2}}{x}-\frac{x u}{3}\right)+D_{x}^{2 \beta} u^{2}\right]\right] .
$$


TABLE 1: Several approximate values and exact solutions for (36) and (37) when $\alpha=\beta=1$.

\begin{tabular}{cccccc}
\hline$x$ & $t$ & Solution $_{\text {ILTM }}$ & Solution $_{\text {HPM }}$ & Solution $_{\text {ADM }}$ & Exact solution \\
\hline \multirow{3}{*}{0.25} & 0.1 & 0.0690729 & 0.0690729 & 0.0690729 & 0.0690732 \\
& 0.2 & 0.0763333 & 0.0763333 & 0.0763333 & 0.0763377 \\
& 0.4 & 0.0931667 & 0.0931667 & 0.0931667 & 0.093239 \\
0.5 & 0.8 & 0.137833 & 0.137833 & 0.137833 & 0.139096 \\
\hline \multirow{3}{*}{0.1} & 0.276292 & 0.276292 & 0.276292 & 0.276293 \\
& 0.2 & 0.305333 & 0.305333 & 0.305333 & 0.305351 \\
0.75 & 0.4 & 0.372667 & 0.372667 & 0.372667 & 0.372956 \\
& 0.8 & 0.551333 & 0.551333 & 0.551333 & 0.556385 \\
\hline & 0.1 & 0.621656 & 0.621656 & 0.621656 & 0.687 \\
\end{tabular}

Following the algorithm given in (17), the first few components of the solution are as follows:

$$
\begin{aligned}
& u_{0}(x, t)=x^{2}, \\
& u_{1}(x, t)=\mathscr{L}^{-1}\left[\frac{1}{s^{\alpha}} \mathscr{L}\left[-D_{x}^{\beta}\left(\frac{4 u_{0}^{2}}{x}-\frac{x u_{0}}{3}\right)+D_{x}^{2 \beta} u_{0}^{2}\right]\right] \\
& =\left(-\frac{22}{\Gamma(4-\beta)} x^{3-\beta}+\frac{24}{\Gamma(5-2 \beta)} x^{4-2 \beta}\right) \\
& \frac{t^{\alpha}}{\Gamma(1+\alpha)}, \\
& u_{2}(x, t) \\
& =\mathscr{L}^{-1}\left[\frac { 1 } { s ^ { \alpha } } \mathscr { L } \left[-D_{x}^{\beta}\left(\frac{4\left(u_{0}+u_{1}\right)^{2}}{x}-\frac{x\left(u_{0}+u_{1}\right)}{3}\right)\right.\right. \\
& \left.\left.+D_{x}^{2 \beta}\left(u_{0}+u_{1}\right)^{2}\right]\right] \\
& -\mathscr{L}^{-1}\left[\frac{1}{s^{\alpha}} \mathscr{L}\left[-D_{x}^{\beta}\left(\frac{4 u_{0}^{2}}{x}-\frac{x u_{0}}{3}\right)+D_{x}^{2 \beta} u_{0}^{2}\right]\right] \\
& =-\frac{\Gamma(1+2 \alpha) \Gamma(8-4 \beta)}{\Gamma^{2}(1+\alpha) \Gamma(1+3 \alpha) \Gamma(8-5 \beta) \Gamma(5-2 \beta)} \\
& \cdot\left(\frac{2304}{\Gamma(5-2 \beta)}+\frac{1056}{\Gamma(4-\beta)}\right) t^{3 \alpha} x^{7-5 \beta} \\
& -\frac{1}{\Gamma(1+2 \alpha) \Gamma(6-3 \beta)}\left(\frac{184 \Gamma(6-2 \beta)}{\Gamma(5-2 \beta)}+\frac{44 \Gamma(6-\beta)}{\Gamma(4-\beta)}\right) \\
& \cdot t^{2 \alpha} x^{5-3 \beta} \\
& +\frac{\Gamma(1+2 \alpha)}{\Gamma^{2}(1+\alpha) \Gamma(1+3 \alpha) \Gamma(7-4 \beta) \Gamma(4-\beta)} \\
& \times\left(\frac{484 \Gamma(7-2 \beta)}{\Gamma(4-\beta)}+\frac{4224 \Gamma(7-3 \beta)}{\Gamma(5-2 \beta)}\right) \cdot t^{3 \alpha} x^{6-4 \beta}
\end{aligned}
$$

$$
\begin{aligned}
& +\frac{506 \Gamma(5-\beta)}{3 \Gamma(1+2 \alpha) \Gamma(5-2 \beta) \Gamma(4-\beta)} \cdot t^{2 \alpha} x^{4-2 \beta} \\
& -\frac{1936 \Gamma(1+2 \alpha) \Gamma(6-2 \beta)}{\Gamma^{2}(1+\alpha) \Gamma(1+3 \alpha) \Gamma(6-3 \beta) \Gamma^{2}(4-\beta)} \cdot t^{3 \alpha} x^{5-3 \beta} \\
& +\frac{576 \Gamma(1+2 \alpha) \Gamma(9-4 \beta)}{\Gamma^{2}(1+\alpha) \Gamma(1+3 \alpha) \Gamma(8-5 \beta) \Gamma^{2}(5-2 \beta)} \cdot t^{3 \alpha} x^{8-6 \beta} \\
& +\frac{48 \Gamma(7-2 \beta)}{\Gamma(1+2 \alpha) \Gamma(7-4 \beta) \Gamma(5-2 \beta)} \cdot t^{2 \alpha} x^{6-4 \beta} \\
& +\left(\frac{22}{\Gamma(4-\beta)} x^{3-\beta}-\frac{24}{\Gamma(5-2 \beta)} x^{4-2 \beta}\right) \cdot \frac{t^{\alpha}}{\Gamma(1+\alpha)}, \\
& \vdots
\end{aligned}
$$

The solution in series form is then given by

$$
u(x, t)=u_{0}(x, t)+u_{1}(x, t)+u_{2}(x, t)+\cdots .
$$

If we take $\alpha=\beta=1$, the first few components of the solution are as follows:

$$
\begin{gathered}
u_{0}(x, t)=x^{2}, \\
u_{1}(x, t)=x^{2} t \\
u_{2}(x, t)=\frac{x^{2} t^{2}}{2},
\end{gathered}
$$

For this special case, the exact solution of (36) and (37) is therefore given by

$$
u(x, t)=x^{2} e^{t}
$$

which is exactly the result obtained by homotopy perturbation transformation method in [12]. 
Table 1 shows the numerical solutions for (36) and (37) by using iterative Laplace transform method, homotopy perturbation transform method, Adomian decomposition method, and the exact solution as $\alpha=\beta=1$. It should be pointed out that only three terms of these methods are used to evaluate the numerical solutions in Table 1 . It is obvious that the iterative Laplace transform method used in the present paper has the same convergence as the convergence of homotopy perturbation transform method and Adomian decomposition method for solving this fractional nonlinear Fokker-Planck equation. Therefore, iterative Laplace transform method is an effective method for solving fractional partial differential equations just as homotopy perturbation transform method and Adomian decomposition method.

\section{Conclusions}

With the aid of the symbolic computation system Mathematica, the iterative Laplace transform method is first successfully applied to solve fractional Fokker-Planck equations. The results obtained by the iterative Laplace transform method are the same as those obtained by homotopy perturbation transform method and Adomian decomposition method. The method finds the solutions without unnecessary linearization, small perturbation and other restrictive assumptions. Therefore, the method considerably reduces the computational work to a great extent. It is worth mentioning that the method can also be applied to solve other nonlinear fractional differential equations with initial value conditions.

\section{Acknowledgments}

The author would like to express her sincere thanks to the referees for their valuable suggestions which lead to an improved version. This research is supported by Excellent Young Scientist Foundation of Shandong Province under Grant no. BS2013HZ026.

\section{References}

[1] J. Sabatier, O. P. Agrawal, and J. A. Tenreiro Machado, Advances in Fractional Calculus: Theoretical Developments and Applications in Physics and Engineering, Springer, 2007.

[2] D. Baleanu, K. Diethelm, E. Scalas, and J. J. Trujillo, Fractional Calculus, vol. 3 of Series on Complexity, Nonlinearity and Chaos, World Scientific, Singapore, 2012.

[3] Y. Liu and B. Xin, "Numerical solutions of a fractional predatorprey system," Advances in Difference Equations, vol. 2011, Article ID 190475, 11 pages, 2011.

[4] J. Ma and Y. Liu, "Exact solutions for a generalized nonlinear fractional Fokker-Planck equation,” Nonlinear Analysis: Real World Applications, vol. 11, no. 1, pp. 515-521, 2010.

[5] M. Rehman and R. Ali Khan, "The Legendre wavelet method for solving fractional differential equations," Communications in Nonlinear Science and Numerical Simulation, vol. 16, no. 11, pp. 4163-4173, 2011.

[6] Y. Li, "Solving a nonlinear fractional differential equation using Chebyshev wavelets," Communications in Nonlinear Science and Numerical Simulation, vol. 15, no. 9, pp. 2284-2292, 2010.
[7] J. L. Wu, "A wavelet operational method for solving fractional partial differential equations numerically," Applied Mathematics and Computation, vol. 214, no. 1, pp. 31-40, 2009.

[8] Ü. Lepik, "Solving fractional integral equations by the Haar wavelet method," Applied Mathematics and Computation, vol. 214, no. 2, pp. 468-478, 2009.

[9] V. S. Ertürk and S. Momani, "Solving systems of fractional differential equations using differential transform method," Journal of Computational and Applied Mathematics, vol. 215, no. 1, pp. 142-151, 2008.

[10] Y. Li and N. Sun, "Numerical solution of fractional differential equations using the generalized block pulse operational matrix," Computers \& Mathematics with Applications, vol. 62, no. 3, pp. 1046-1054, 2011.

[11] A. Saadatmandi and M. Dehghan, "A new operational matrix for solving fractional-order differential equations," Computers \& Mathematics with Applications, vol. 59, no. 3, pp. 1326-1336, 2010.

[12] Y. Liu, "Approximate solutions of fractional nonlinear equations using homotopy perturbation transformation method," Abstract and Applied Analysis, vol. 2012, Article ID 752869, 14 pages, 2012.

[13] Y. Liu, "Variational homotopy perturbation method for solving fractional initial boundary value problems," Abstract and Applied Analysis, vol. 2012, Article ID 727031, 10 pages, 2012.

[14] N. H. Sweilam, M. M. Khader, and R. F. Al-Bar, "Numerical studies for a multi-order fractional differential equation," Physics Letters A, vol. 371, no. 1-2, pp. 26-33, 2007.

[15] S. Das, "Analytical solution of a fractional diffusion equation by variational iteration method," Computers \& Mathematics with Applications, vol. 57, no. 3, pp. 483-487, 2009.

[16] H. Jafari, C. M. Khalique, and M. Nazari, "Application of the Laplace decomposition method for solving linear and nonlinear fractional diffusion-wave equations," Applied Mathematics Letters, vol. 24, no. 11, pp. 1799-1805, 2011.

[17] M. Y. Ongun, “The Laplace adomian decomposition method for solving a model for HIV infection of $\mathrm{CD} 4^{+}$cells," Mathematical and Computer Modelling, vol. 53, no. 5-6, pp. 597-603, 2011.

[18] V. Daftardar-Gejji and H. Jafari, "An iterative method for solving nonlinear functional equations," Journal of Mathematical Analysis and Applications, vol. 316, no. 2, pp. 753-763, 2006.

[19] H. Jafari, Iterative Methods for solving system of fractional differential equations [Ph.D. thesis], Pune University, 2006.

[20] S. Bhalekar and V. Daftardar-Gejji, "Solving evolution equations using a new iterative method," Numerical Methods for Partial Differential Equations, vol. 26, no. 4, pp. 906-916, 2010.

[21] V. Daftardar-Gejji and S. Bhalekar, "Solving fractional boundary value problems with Dirichlet boundary conditions using a new iterative method," Computers \& Mathematics with Applications, vol. 59, no. 5, pp. 1801-1809, 2010.

[22] H. Jafari, M. Nazari, D. Baleanu, and C. M. Khalique, "A new approach for solving a system of fractional partial differential equations," Computers \& Mathematics with Applications, vol. 66, no. 5, pp. 838-843, 2013.

[23] H. Jafari and S. Seifi, "Solving a system of nonlinear fractional partial differential equations using homotopy analysis method," Communications in Nonlinear Science and Numerical Simulation, vol. 14, no. 5, pp. 1962-1969, 2009.

[24] H. Risken, The Fokker-Planck Equation, Methods of Solution and Applications, Springer, Berlin, Germany, 1989. 
[25] T. D. Frank, "Stochastic feedback, nonlinear families of Markov processes, and nonlinear Fokker-Planck equations," Physica A, vol. 331, no. 3-4, pp. 391-408, 2004.

[26] A. Yiidirim, "Analytical approach to Fokker-Planck equation with space- and time fractional derivatives by means of the homotopy perturbation method," Journal of King Saud University, vol. 22, no. 4, pp. 257-264, 2010.

[27] K. S. Miller and B. Ross, An Introduction to the Fractional Calculus and Fractional Differential Equations, John Wiley \& Sons, New York, NY, USA, 1993.

[28] I. Podlubny, Fractional Differential Equations, vol. 198, Academic Press, New York, NY, USA, 1999.

[29] R. Hilfer, Applications of Fractional Calculus in Physics, World Scientific, Singapore, 2000.

[30] J. Biazar, K. Hosseini, and P. Gholamin, "Homotopy perturbation method Fokker-Planck equation," International Mathematical Forum, vol. 3, no. 19, pp. 945-954, 2008. 


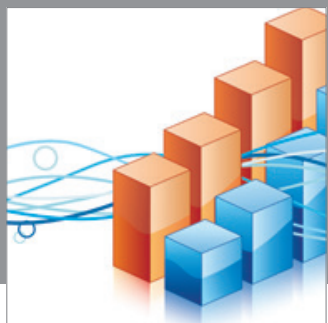

Advances in

Operations Research

mansans

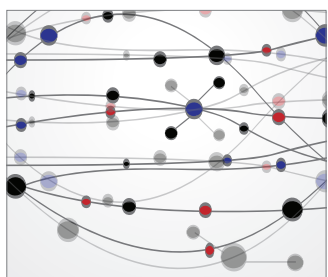

The Scientific World Journal

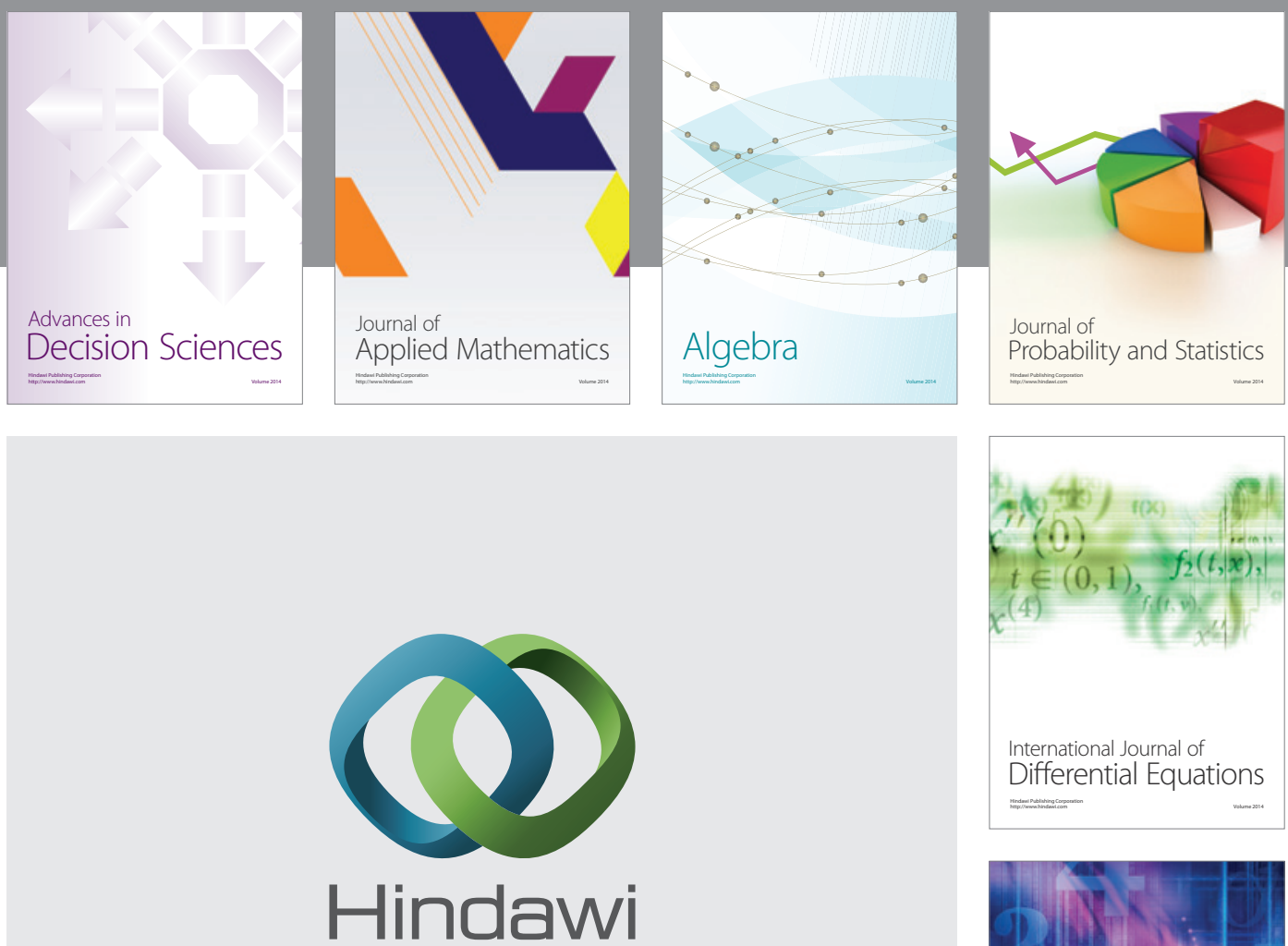

Submit your manuscripts at http://www.hindawi.com
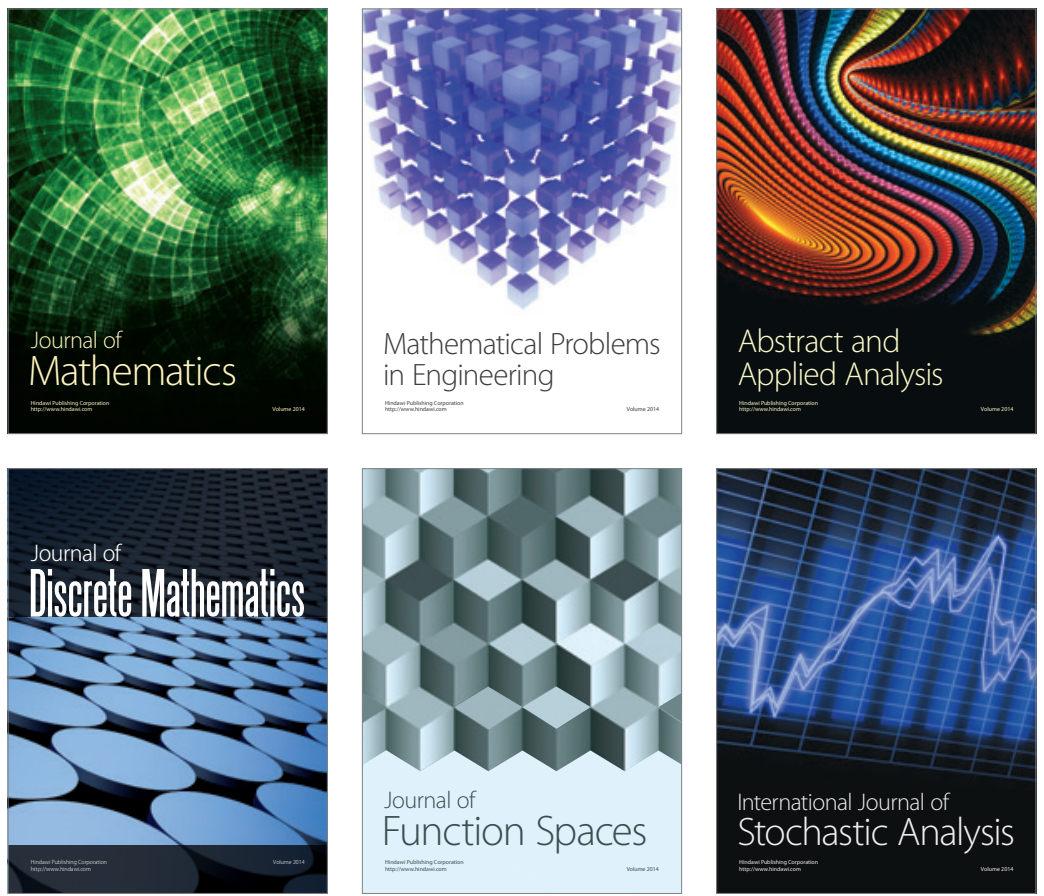

Journal of

Function Spaces

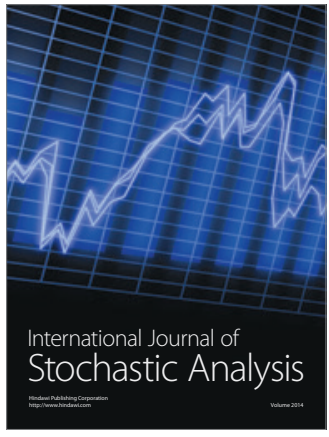

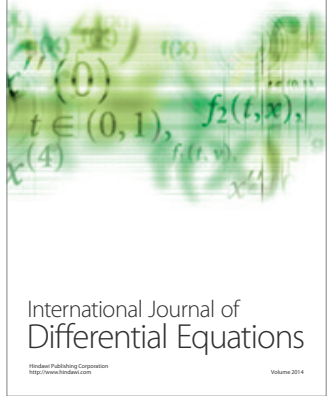
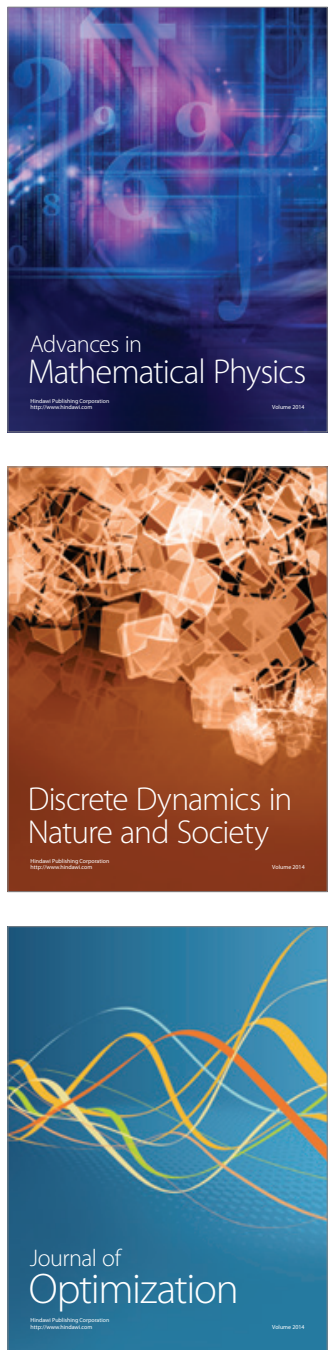